\title{
Analysis of Jitter Peaking Effects in Digital Long-Haul Transmission Systems Using SAW-Filter Retiming
}

\author{
DANIEL A. FISHMAN, ROBERT L. ROSENBERG, SENIOR MEMBER, IEEE, AND \\ CHRISTODOULOS CHAMZAS, MEMBER, IEEE
}

\begin{abstract}
The new lightwave long-haul transmission systems typically use surface-acoustic-wave (SAW) filters for timing recovery, in place of the phase-locked loops favored in slower systems. We report here analytical studies of jitter phenomena allowed by two kinds of filter ripple. The ripple is capable of causing jitter to accumulate exponentially with the number of regenerators $N$ in a repeatered line. Such behavior is well known in the case of phase-locked-loop retiming, where the "jitter peaking" that usually appears in the loop response must be carefully limited to avoid exponential jitter growth. We show that equivalent phenomena can appear when the SAW filters exhibit passband ripple, or, as previously reported in condensed form, when ripple-free filters are detuned by approximately one-half the full $3 \mathrm{~dB}$ bandwidth. Furthermore, in the case of ripply filters, exponential jitter accumulation is found to be much more pronounced for random jitter than for systematic jitter. In addition, the alignment jitter within each regenerator can grow exponentially along the chain of regenerators. Neither of these statements is true in the case of the ripple-free filters previously treated in the literature.
\end{abstract}

\section{INTRODUCTION}

L IGHTWAVE transmission systems currently being deJveloped may utilize as many as 200 regenerators in the case of undersea systems. The jitter characteristics of these chains are of considerable interest because of the new technologies being applied. This study treats, in particular, the accumulation of line jitter when SAW filters are used for timing recovery in the regenerator timing loop. In fiber systems, these passive filters are favored over phase-locked loops, whose implementation has proved difficult at the high signaling rates of interest $(>100 \mathrm{Mbits} / \mathrm{s})$. Two types of SAW filters have been candidates for the timing recovery task [1], [2], the double resonator type, analogous to LC double-tuned filters, and the transversal type, which is nonminimum phase. Transversal filters are easier to implement at lower $Q$ values, while resonator filters are easier at higher $Q$ values [3]. For various reasons, the $Q$ 's needed for retiming of uncoded traffic have tended to lie in a transition region between the two types, e.g., near 800 or somewhat higher [2] .

Application of these newer filter types to timing recovery in long-haul digital transmission systems raises questions that have received scant attention in the literature. Some of the questions are examined. here. Perhaps the single most important matter is the possibility of jitter peaking in the jitter transfer function of the filter. Jitter peaking is the occurrence of an absolute maximum in the modulus of the jitter transfer function at a nonvanishing frequency displacement from the baud frequency. This phenomenon is well known in the case of phase-locked loop retiming [4], [5], where it may lead to exponential timing jitter accumulation in the downstream part of a long repeatered line. The analogous passive-filter phenomenon is explored here for two potential

Paper approved by the Editor for Fiber Optics of the IEEE Communications Society for publication without oral presentation. Manuscript received November 7, 1984; revised January 31, 1985.

The authors are with AT\&T Bell Laboratories, Holmdel, NJ 07733. sources of jitter peaking, ripple on the filter passband, and detuning of the filter center frequency with respect to the baud frequency as a result of various mechanisms [2]. For ideal resonator filters, detuning is found to be ignorable as a cause of jitter peaking under normal operating conditions, as reported briefly in [2]. Passband ripple, on the other hand, is a real hazard. In transversal filters employing bidirectional transducers, passband ripple is commonly produced by interference effects traceable to multiple transducer reflections, which become stronger as the transduction efficiency is improved through impedance matching. Therefore, the insertion loss of these filters can typically be lowered only at the expense of increasing the passband ripple. In doubleresonator filters, passband ripple of the Chebyshev type is possible with insufficient impedance matching of the input and output transducers. The jitter accumulation effects of both passband ripple and static filter detuning are demonstrated in the present work.

In Section II and the Appendix, we describe the model used to represent the SAW filters. Analytic expressions for computing the random and systematic jitter accumulations and alignment jitter are presented in Section III. In Section IV, we review some relations for treating the effects of resonator-filter detuning on jitter accumulation. The analytical tools are applied in Section $\mathrm{V}$ to a survey of ripple and detuning effects on jitter accumulation and alignment jitter. A brief summary appears in Section VI.

\section{SAW FiLTER MODELING}

Our approach requires a filter transfer function to compute the jitter accumulation caused by self-timed regenerators. In this section we discuss the two filter types of potential interest and give their transfer functions.

\section{A. Double Resonator Filter}

The double-resonator SAW filter has a resonance line shape (power spectrum) of the form [6]

$$
P(x)=\frac{1}{1+\left\{\frac{(q x)^{2}-(q k)^{2}+1}{2 q k}\right\}^{2}}
$$

where $q \equiv Q_{\text {loaded }} / Q_{F}, Q_{\text {loaded }}$ is the loaded $Q$ of each resonator, $Q_{F}=f_{c} / 2 f_{3 \mathrm{~d} \mathrm{~B}}, x=\left(f-f_{c}\right) / f_{3 \mathrm{~d} \mathrm{~B}}, k$ is the interresonator coupling coefficient, $f_{c}$ is the filter center frequency, and $f_{3 \mathrm{~dB}}$ is half the $3 \mathrm{~dB}$ passband width of the filter. For present purposes, the cases of interest are $q k=1$ and $q k>1$, corresponding to maximally flat (Butterworth) and ripply (Chebyshev) filters, respectively. The corresponding phase relationship is [7]

$$
\phi=\tan ^{-1}\left\{\frac{-2 q x}{\left(k^{2} q^{2}-q^{2} x^{2}\right)+1}\right\} .
$$

For the Butterworth case, $q=\sqrt{2}, k=1 / \sqrt{2}$, and $P(x)$ 
reduces to $1 /\left(1+x^{4}\right)$. In terms of frequency variables, we may write

$$
P\left(f-f_{c}\right)=\frac{1}{1+\left[\frac{f-f_{c}}{f_{3 \mathrm{~d} \mathrm{~B}}}\right]^{4}}
$$

where $P(f)$ is the absolute square of the filter transfer function [5]

$$
H(f)=\frac{1}{1+j \sqrt{2} f / f_{3 \mathrm{~d} \mathrm{~B}}-\left(f / f_{3 \mathrm{~dB}}\right)^{2}} .
$$

All of these relations omit the effects of frequency variation of the piezoelectric transduction process [6]. The omission is justifiable across the upper passband [6]. A more complete model is not needed here.

The case of the Chebyshev filters $(k>1 / \sqrt{2})$ corresponds to designs with symmetric passband ripple. The double-resonator filter transfer function for a Butterworth filter and for a Chebyshev filter with $0.5 \mathrm{~dB}$ ripple are shown in Fig, 1. A single-tuned filter characteristic [7] is shown for comparison. Double-resonator SAW filters in digital transmission spans should be of the maximally flat or single-peaked type $(k<1 / \sqrt{2})$ if one wishes to avoid jitter peaking.

\section{B. Transversal SAW Filter}

While the double-resonator filter is an infinite-impulseresponse (IIR) device with a characteristic nonlinear phase, the transversal filter is a finite-impulse-response (FIR) device with a nearly linear phase [8] dominated by transmission delay. Since a transversal filter is a nonminimum phase device, ${ }^{1}$ virtually any amplitude characteristic can be approximated to first order by using enough delay elements and taps. With a SAW realization, the ideal response is perturbed by a number of different effects. The most important perturbation for present purposes is the triple transit response, caused by unwanted SAW reflections from the receiving and transmitting transducers in turn. The triple transit signal causes a ripple in the amplitude and phase versus frequency characteristics. The amplitude ripple is of primary importance here.

A simple description of a SAW transversal filter that exhibits the essentials of filter behavior and includes triple transits is developed in the Appendix and summarized below. The approximate filter transfer function, including multiple transits, is

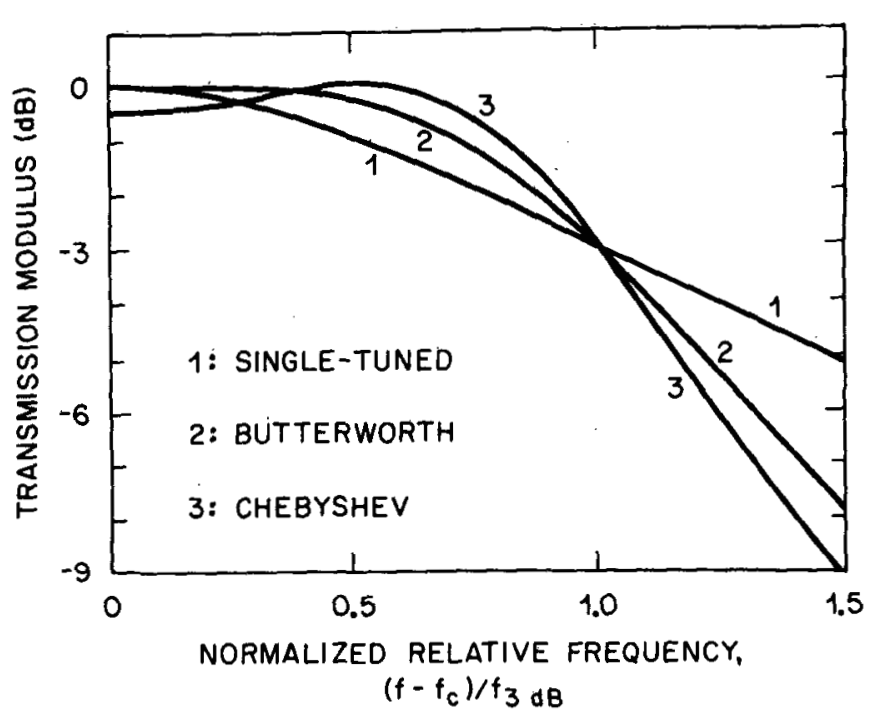

(a)

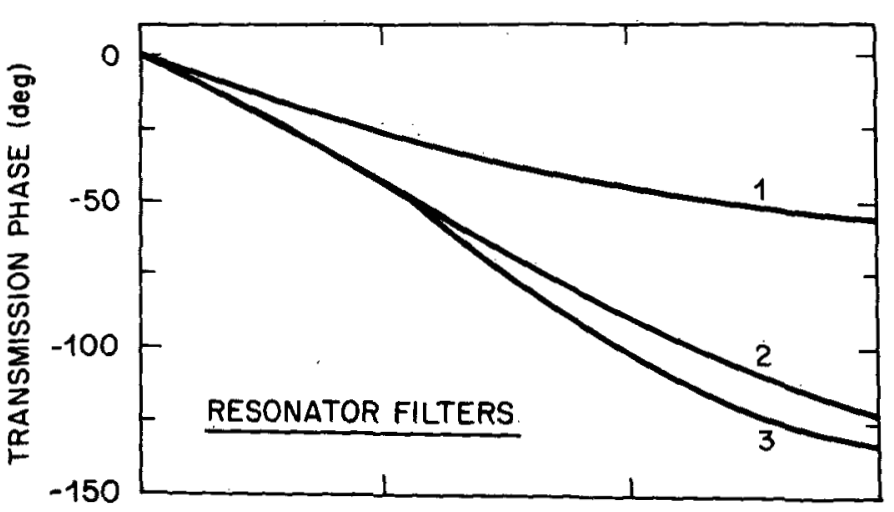

(b)

Fig. 1. (a) Filter moduli for SAW single-tuned and double-tuned resonator filters. Across the passband, these idealized curves are indistinguishable from those of analogous RLC filters. (b) Phase-frequency characteristics of the same filters. The frequency responses of transducers and couplers have been neglected.

$\beta d=2 \pi\left(N_{T}+n\right)+2 x\left(1+\frac{n}{N_{T}}\right)$

$$
H(\omega)=\frac{-2 \mu E e^{-j \beta d} e^{-j \psi}}{\left\{\left[(1+\mu E)^{2}-(\mu O)^{2}-(\mu E)^{2} \cos 2 \beta d\right]^{2}+\left[2 \mu O(1+\mu E)+(\mu E)^{2} \sin 2 \beta d\right]^{2}\right\}^{1 / 2}}
$$

where

$$
\begin{aligned}
& \psi=\tan ^{-1}\left\{\frac{2 \mu O(1+\mu E)+(\mu E)^{2} \sin 2 \beta d}{(1+\mu E)^{2}-(\mu O)^{2}-(\mu E)^{2} \cos 2 \beta d}\right\} \\
& x=\pi N_{T}\left(\frac{\omega}{\omega_{c}}-1\right)
\end{aligned}
$$

\footnotetext{
1 The transfer function of an ideal transversal filter is written as a single polynomial $A(\omega)=\sum_{n=1}^{N} a_{n} \exp \left(i \omega T_{n}\right)$, where $N$ is the number of taps, $a_{n}$ is the tap strength, and $T_{n}$ is the delay between the input and the $n$th tap. Since there are no poles, the pole-zero minimum phase filter design techniques cannot be used.
}

$$
\begin{aligned}
& O(\omega)=\frac{-1}{x}\left(1-\frac{\sin 2 x}{2 x}\right) \\
& E(\omega)=\left\{\frac{\sin x}{x}\right\}^{2}
\end{aligned}
$$

with

$$
\begin{aligned}
\omega_{c}= & \text { center radian frequency } \\
N_{T}= & \text { number of finger periods in each transducer } \\
n= & \text { number of periods at } \omega_{c} \text { between transducers, } \\
& \text { not necessarily an integer }(n \approx 20 \text { is reasonable }) .
\end{aligned}
$$

In the above expressions, $\mu$ is an admittance mismatch param- 
eter defined by

$$
\mu=\frac{G_{a}\left(\omega_{c}\right)}{G_{L}}
$$

where $G_{a}\left(\omega_{c}\right)$ is the acoustic radiation conductance of each transducer at center frequency; and $G_{L}$ is the conductance level of the adjacent circuits, e.g., $(50 \Omega)^{-1}$. For present purposes, $\mu$ is regarded as adjustable through known design methods. For filters fabricated on a quartz substrate, $\mu$ is normally $<1$, and is often $\ll 1$. Multiple transit terms are those in $\sin (2 \beta d)$ and $\cos (2 \beta d)$. The multiple transit terms produce a local dip at center frequency when $N_{T}+n=$ integer +0.25 , so that

$$
\begin{aligned}
& \sin 2 \beta_{c} d=0 \\
& \cos 2 \beta_{c} d=-1 \\
& \sin 2 \beta d=-\sin \left[4 x\left(1+\frac{n}{N_{T}}\right)\right] \\
& \cos 2 \beta d=-\cos \left[4 x\left(1+\frac{n}{N_{T}}\right)\right]
\end{aligned}
$$

This is a "worst case" for jitter accumulation.

The above formulation leads to a convenient representation of amplitude ripple as follows. By means of (4a) and (4c), the amplitude characteristic is expressible as a function of $x$ and three parameters, $\mu, n$, and $N_{T}$. Of these parameters, only $\mu$ is allowed to vary independently. For practical reasons discussed in the Appendix, $n$ is set equal to 20.25 (symmetric ripple). $N_{T}$ is determined from $\mu$ and a preselected value of $Q$ (e.g., 800), through the relations discussed in the Appendix:

$$
N_{T}=2 Q y(\mu) / \pi
$$

where $y(\mu)$ is the solution of (35), which locates the $3 \mathrm{~dB}$ points of the ripple-free filter transmission. With $n$ and $N_{T}$ known, the ripple corresponding to a given value of $\mu$ is determined by computing the function $|H(x)|^{2}$. Compatible values of ripple and $N_{T}$ are obtained in this way for a range of values of $\mu$. The transfer function is then completely characterized for present purposes. Sample results are plotted in Fig. 2, which provides parameters of the model associated with arbitrary ripple magnitude. The dashed lines show how one proceeds from a specific value of ripple to a corresponding value of conductance mismatch $\mu$, and thence to the associated number of transducer periods $N_{T}$ via $y(\mu)$ and a given $Q$.

More generally, the above expressions can be used to obtain filter transfer functions of the sort shown in Fig. 3. Magnitude characteristics are shown in Fig. 3(a) for the case of a central dip (worst-case ripple, $N_{T}+n-$ integer $=0.25$ ), a central maximum $\left(N_{T}+n-\right.$ integer $\left.=0\right)$, and an asymmetric case $\left(N_{T}+n\right.$ - integer $\left.=0.125\right)$, all computed with other parameters held fixed. The characteristic obtained by neglecting multiple transits is included for comparison. Associated phase characteristics are show in Fig. 3(b).

\section{JitTer Accumulation Model}

\section{A. Chapman Model}

Jitter accumulation will be formulated here in accord with the well-known Chapman model [9] illustrated in Fig. 4. In the Chapman model, all regenerators in the transmission channel are assumed to have identical characteristics, described by a complex "jitter transfer function" $W(f) . W(f)$ differs

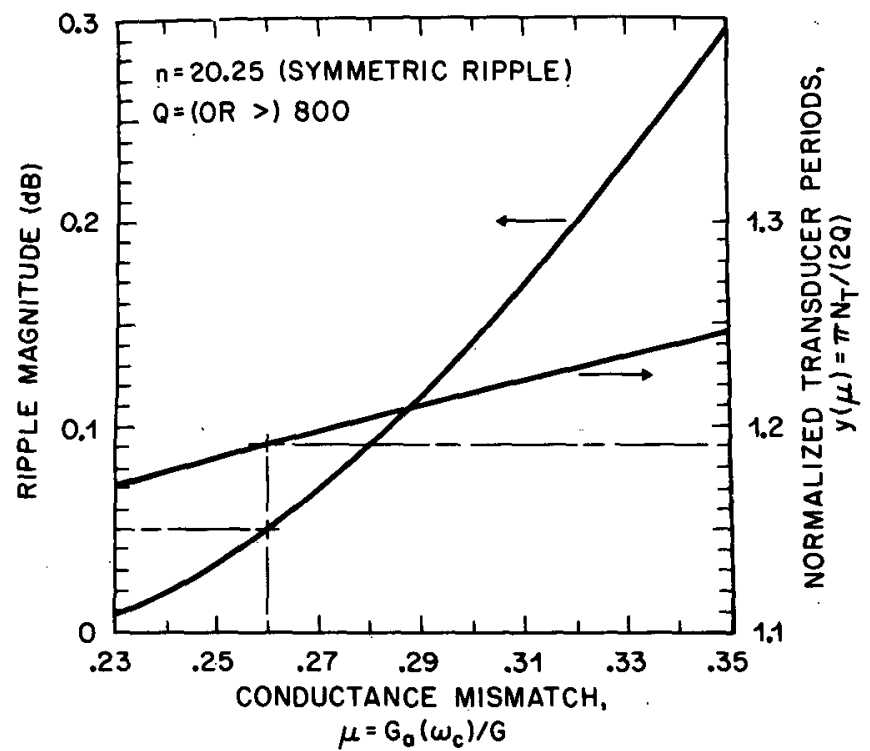

Fig. 2. Curves relating the filter parameters of interest for studying ripple effects with the present transversal filter model.

from the filter transfer function $H(f)$ described above in two fundamental ways. First, the jitter transfer function is a lowpass function obtained by shifting the baud frequency to zero. Second, $W(f)$ is a specially symmetrized transform of the low-pass version $H_{\mathrm{L}, \mathrm{P}}(f)$ of the filter transfer function [10]. $W(f)$ and $H_{\mathrm{LP}}(f)$ are effectively the same when the modulus of $H_{\mathrm{LP}}(f)$ is an even function of $f$ and the phase of $H_{\mathrm{LP}}$ is an odd function of $f[10]$. When these symmetry conditions prevail, the ripple magnitude of a filter transfer function is effectively the "jitter peak" magnitude of the jitter transfer function. (Other circuit components are broadband compared to the retiming filter, and have little effect on $W(f)$, except through filter loading.) Most of the computations reported here employ filter transfer functions with the above symmetry properties. This approach has the double advantage of representing the "worst-case" jitter while keeping the analysis and computation as simple as possible. A generalization will be introduced when needed to treat the case of detuned filters.

We note that the use of a transfer function implies a linear relationship between the input and output phase deviations. The linear assumption could be questioned on the grounds that passive filtering is intrinsically nonlinear in the phase fluctuations. For the rather small jitter peaking (a few tenths of a decibel) of interest for long-haul systems currently in development, however, estimates show [10] that linearized computations are effectively correct.

As shown in Fig. 4, the Chapman model also assumes that the jitter contributed by each regenerator adds to the incoming jitter at the regenerator input. The added jitter at the $k$ th regenerator is represented by a phase density variable $\theta_{k}(\omega)$, with units of degrees $/ \sqrt{\mathrm{Hz}}$, for example. Because of the additive nature of the jitter, $\theta_{1}(\omega)$ is filtered by all $N$ regenerators, $\theta_{2}(\omega)$ is filtered by $N-1$ transfer functions, and so on. The total jitter at the end of the chain can thus be written in well-known terms [9], [5]: the jitter magnitude $\Theta_{N}(\omega)$ is the sum of all contributions,

$$
\Theta_{N}(\omega)=\sum_{k=1}^{N} \theta_{k}(\omega)[w(\omega)]^{N-k+1}
$$

and the mean jitter "power" density at the end of the chain is

$$
s_{N}(\omega)=\left\langle\left|\Theta_{N}(\omega)\right|^{2}\right\rangle
$$



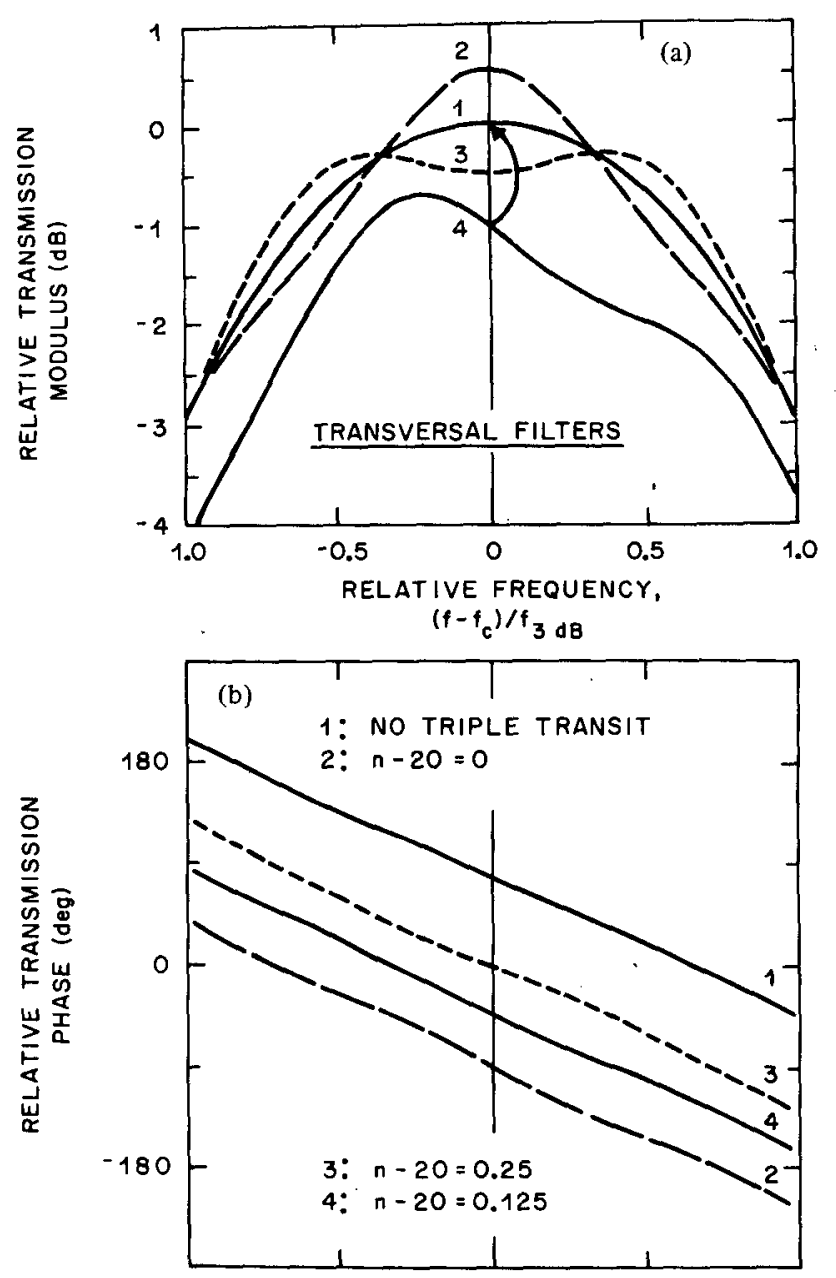

Fig. 3. (a) SAW transversal-filter transmission moduli showing various passband shapes derived from the model. Triple-transit interference is highly sensitive to fractional-wavelength changes in the transducer separation. As indicated by the arrow, curve 4 for the asymmetric filter has been shifted $1 \mathrm{~dB}$ downward from its correct relative position, for clarity. (b) Phase-frequency characteristics of the same filters. Curves 2-4 are shown in correct relative positions. Curve 1 also depends on $n$, and can cross the vertical axis anywhere.

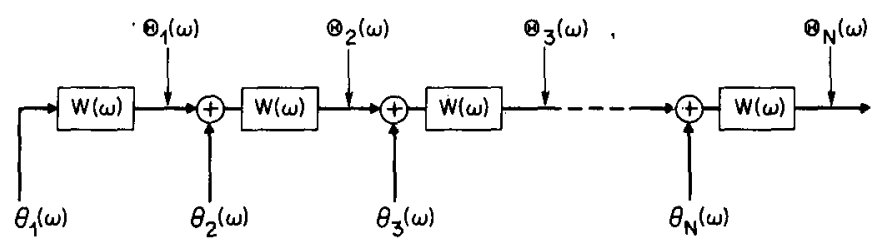

Fig. 4. The Chapman model for jitter accumulation. The jitter transfer function $W(\omega)$ is the same for all regenerators. The timing (phase) jitter $\theta_{k}(\omega)$ added at the $k$ th regenerator is the same for all $k$ in the case of systematic (pattern-dependent) jitter, and is an independent random variable in the case of random jitter (phase noise). Accumulated jitter after $\boldsymbol{k}$ regenerators is represented by $\boldsymbol{\theta}_{\boldsymbol{k}}(\omega)$.

where the corner brackets signify ensemble averages taken over all random variables. Two jitter types are customarily distinguished. Systematic jitter is caused by variations in the message pattern itself. Since the digital message is the same at every regenerator, barring errors, the added systematic jitter will be the same at every regenerator and can be represented by a common random variable $\theta_{s}(\omega)=\theta_{k}(\omega)$. Then from (8) and (9), the systematic jitter power density $S_{N, s}(\omega)$ contains a coherent sum of jitter transfer functions,

$$
\begin{aligned}
S_{N, s} & =S_{S}(\omega)\left|\sum_{k=1}^{N}[W(\omega)]^{N-k+1}\right|^{2} \\
& =S_{s}(\omega)|W(\omega)|^{2}\left|\frac{1-W(\omega)^{N}}{1-W(\omega)}\right|^{2}
\end{aligned}
$$

where $S_{s}(\omega)=\left\langle\left|\theta_{s}^{2}(\omega)\right|\right\rangle$. On the other hand, random jitter is caused by noise, which requires a distinct random variable at each regenerator. Although the variables are independent, they are assumed to have the same statistical properties, so that after averages in (9) are evaluated, the random jitter "power" density $S_{N, r}(\omega)$ may be written in a form analogous to $(10)$, where the summation on the transfer functions is now incoherent:

$$
\begin{aligned}
S_{N, r} & =S_{r}(\omega) \sum_{k=1}^{N}|W(\omega)|^{2(N-k+1)} \\
& =S_{r}(\omega)|W(\omega)|^{2}\left\{\frac{1-|W(\omega)|^{2 N}}{1-|W(\omega)|^{2}}\right\}
\end{aligned}
$$

with $S_{r}(\omega)=\left\langle\theta_{r}{ }^{2}(\omega)\right\rangle$, independently of $k$. The mean squared value of the total accumulated jitter is an integral over the jitter densities,

$$
\sigma_{N}^{2}=\frac{1}{2 \pi} \int_{-\infty}^{\infty}\left[S_{N, s}(\omega)+S_{N, r}(\omega)\right] d \omega .
$$

Typically, the integrand is completely controlled by the jitter transfer function summations, and $S_{s}(\omega)$ and $S_{r}(\omega)$ may be assumed constant at their low-frequency values. The integration can then be carried out in nearly closed form for the Butterworth function (3), as shown in the equivalent case of a particular second-order phase-locked loop [5]. The result is that rms systematic and random jitter accumulate, respectively, as $N^{1 / 2}$ and $N^{3 / 8}$ for a double-resonator Butterworth filter. In the better known case of a single-resonator filter, the corresponding power laws are $N^{1 / 2}$ and $N^{1 / 4}$ [9]. We shall presently show that the accumulation laws can change dramatically when the ripple on the filter passband generates a peak in the jitter transfer function away from zero jitter frequency. (The jitter frequency is equivalent to $\left|f-f_{b}\right|$ where $f_{b}$ is the baud frequency.) All rms values in this paper will be normalized to $\sigma_{1}$, which may represent either systematic or random jitter produced in the first regenerator, according to context.

\section{B. Generalized Chapman Model}

Since systematic jitter accumulation depends on a coherent sum of complex transfer functions, as in (10), one expects the value of the sum to be sensitive to effects that can rephase individual transfer functions. Such an effect actually occurs during the manufacture of transversal filters, which show a distribution of phase slopes. The consequence for messagedependent jitter accumulation can be estimated by randomly distributing the linear part of the phase characteristics of the cascaded regenerators. A model of this type has been evaluated, but details will not be given here. For all cases of practical interest, the associated dephasing effects are too small to merit discussion.

\section{Relative or Alignment Jitter}

While accumulated timing jitter at the end of an $N$-regenerator repeatered line may span many time slots, the effect on the bit error ratio (BER) in the last generator may still be 
negligible. The BER can only be effected by the relative (or alignment) jitter, which measures the offset of data pulse positions relative to the corresponding retiming threshold crossings [11]. Relative jitter causes errors by decreasing the decision eye margins, and must remain bounded to achieve useful where $f_{b}$ is the baud frequency, $\chi$ is the transmission phase offset caused by frequency detuning from the baud, and $t_{0}$ is the corresponding decision-time offset. $H_{L P}(f)$ is a low-pass transfer function, given for simple resonator filters [ [12], [13] by

$$
H_{\mathrm{LP}}(f)= \begin{cases}\frac{1}{1+j 2 Q\left(f / f_{b}-\eta\right)} & \text { (single resonator) } \\ \frac{1}{1+j 2 \sqrt{2} Q\left(f / f_{b}-\eta\right)-\left[2 Q\left(f / f_{b}-\eta\right)\right]^{2}} & \text { (double resonator) }\end{cases}
$$

system operation. Relative jitter is of interest because it will be shown to grow with $N$ when the timing jitter accumulates exponentially.

The formulation of relative jitter follows from its definition as the difference between the output jitter and the input jitter at each regenerator. Since the input jitter comes from the output jitter of the preceding regenerator, relative jitter in the $N$ th regenerator can be calculated from the difference

$$
\delta_{N}(\omega)=\Theta_{N}(\omega)-\Theta_{N-1}(\omega)
$$

where $\Theta_{N}(\omega)$ is the sum (8) of the phase density contributions along the entire chain. The mean alignment jitter power density is then

$$
S_{A N}(\omega)=\left\langle\left|\delta_{N}(\omega)\right|^{2}\right\rangle,
$$

an expression with separate.systematic and random parts. The mean squared alignment jitter is finally found through an integral strictly analogous to (12). Evaluation of (14) yields the following expressions for systematic and random contributions:

$$
\begin{aligned}
S_{A N, s} / S_{S}= & |W|^{2 N} \\
S_{A N, r} / S_{r}= & |W|^{2 N}+|W|^{2}\left(2-W-W^{*}\right) \\
& \cdot \frac{1-|W|^{2(N-1)}}{1-|W|^{2}}
\end{aligned}
$$

where we have again assumed that every regenerator adds random jitter with the same statistical properties. An asterisk denotes complex conjugate. If the second term of the random contribution could be ignored, the systematic and random contributions would have the same behavior. The seciond random term occurs because of an absence of coherence between the incoming random jitter at each regenerator and the random jitter added by that regenerator. Cancellations leading to (15) are therefore inoperative in (16). The extra random term hăs a dramatic effect when jitter peaking occurs.

\section{JitTer ACCUMulation Caúsed by Detuning oR ASYMMETRIC SingLE AND DOUBLE RESONATOR} SAW FILTERS

The approach here is to apply the basic Chapman model with a rederived jitter transfer function that incorporates the detuning effects. Under the assumption that the bit pulses are delta functions, the Mengali and Pirani jitter transfer function [12] can be written

$$
W(f) \approx\left[\frac{H_{\mathrm{LP}}(f) e^{i \chi}-H_{\mathrm{LP}} *(-f) e^{-j \chi}}{H_{\mathrm{LP}}(0) e^{j \chi}-H_{\mathrm{LP}}{ }^{*}(0) e^{-j \chi}}\right]
$$

when $Q \gg 1$. Here,

$$
\chi=2 \pi f_{b} t_{0}
$$

where the detuning parameter is $\eta=\left|f_{c} / f_{b}-1\right|$. Note that even without detuning $(\eta=0)$, the form $(17)$ is useful for the ease of asymmetric passband shape. The jitter accumulates in accord with the Chapman model results (10) and (11) for systematic jitter and random jitter.

Results of detuning studies for single pole filters [9], $[12],[13]$ show that there are two terms in the local jitter spectral densities $S_{s}(f)$ and $S_{r}(f)$, a low-frequency term due to amplitude-to-phase conversion, and a high-frequency term from detuning alone. The low-frequency term increases monotonically along a chain of regenerators, and predominates in long chains. The high-frequency term reaches a limit independent of the length of the regenerator chain [13].

The results from references cited here and those reported in this paper assume that the bit stream can be modeled in terms of delta function pulses. Since some additional lowfrequency jitter is a consequence of the pulse shape alone $[12],[13]$, this assumption may be questionable, especially for NRZ data. To avoid this approximation, the spectral densities $S_{s}(f)$ and $S_{r}(f)$ are assumed to be determined experimentally as in [10]. The assumption of delta function pulses is only used in the derivation of (17), where the effect is found to be minimal. Calculations show that using a $2 T$ raised cosine pulse in the derivation of (17) does not significantly change the jitter characteristics. Furthermore, preliminary experimental measurements verify the results obtained from the present computer model [2].

\section{JitTer ACCumulation Results}

\section{A. Effects of Double-Resonator Filter Detuning and Passband Ripple}

The case of doubled-turied filters will be discussed first, since it relates to previously published work on detuned single-tuned filters [12] and double-tuned filters [2]. The systematic jitter spectral density has been computed from (10) for double-tuned filters detuned by selected amounts. Results are shown in Fig. 5(a) with detuning values given by $2 Q \eta=$ $0.0,0.5$, and 1.15 . Note the arbitrary $10 \mathrm{~dB}$ vertical offsets inserted between curves for clarity. The last value exceeds the approximate threshold of 1.0 [2] for the onset of jitter peaking. The systematic jitter accumulation curves for the same detuning values, computed from (12), are shown in Fig. 5(b). Two general trends are apparent. First, detuning tends to reduce the jitter accumulation by causing a faster rolloff in the jitter transfer modulus $|W(\omega)|$. This trend is apparent over the full curve for $2 Q \eta=0.5$, and over the lower- $N$ part of the curve for $2 Q \eta=1.15$. The second trend dominates for the larget $N$ values on the latter curve. There, exponential jitter growth takes over because detuning is large enough to produce a central dip in the jitter transfer function (i.e., jitter peaking). As pointed out in [2]., such large detuning is inconsistent with good digital error performance, and would never be allowed in designing a transmission system. This source of runaway jitter is therefore of no practical interest.

On the other hand, jitter peaking effects appear without detuning when Chebyshev ripple is present. Fig. 6 shows the 

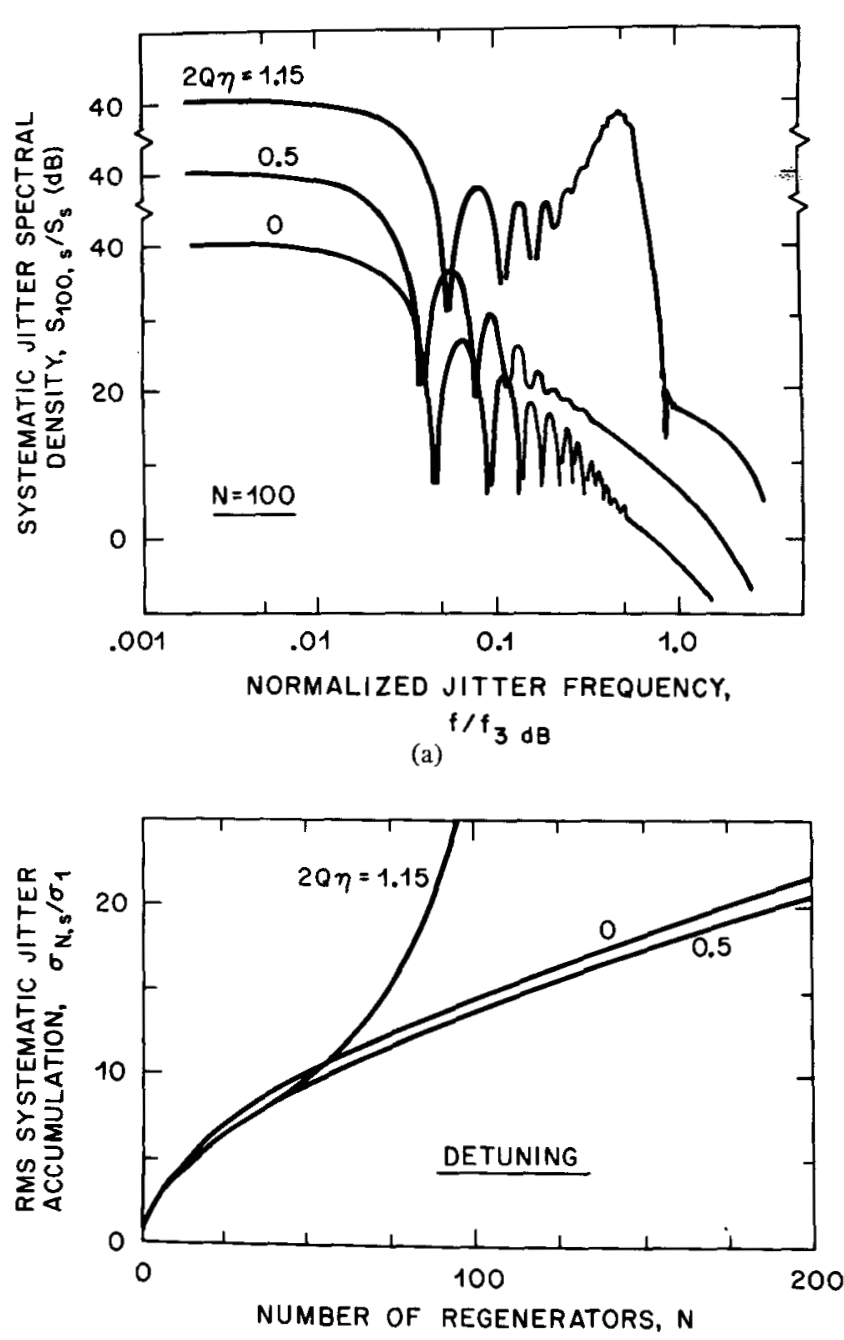

(b)

Fig. 5. (a) Systematic jitter spectral density for Butterworth filters in a cascade of 100 regenerators with selected values of detuning. $\eta=\mid f_{c} / f_{b}$ -11 , where $f_{c}$ and $f_{b}$ are, respectively, the filter center frequency and the baud frequency. $Q$ is the inverse of the $3 \mathrm{~dB}$ fractional bandwidth. The curves are offset for clarity. (b) Rms systematic jitter accumulation for the same filters.

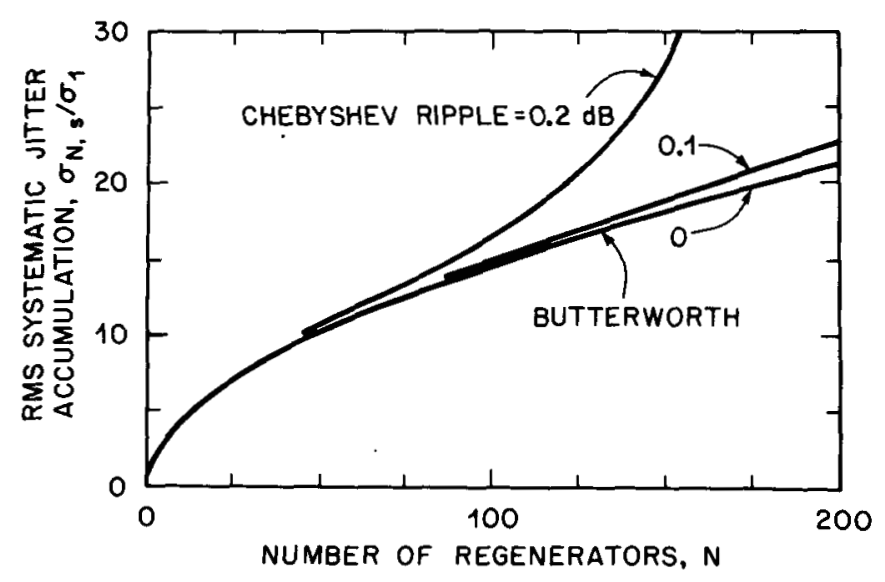

Fig. 6. Systematic jitter accumulation for double-tuned Chebyshev filters with selected passband ripple magnitudes.

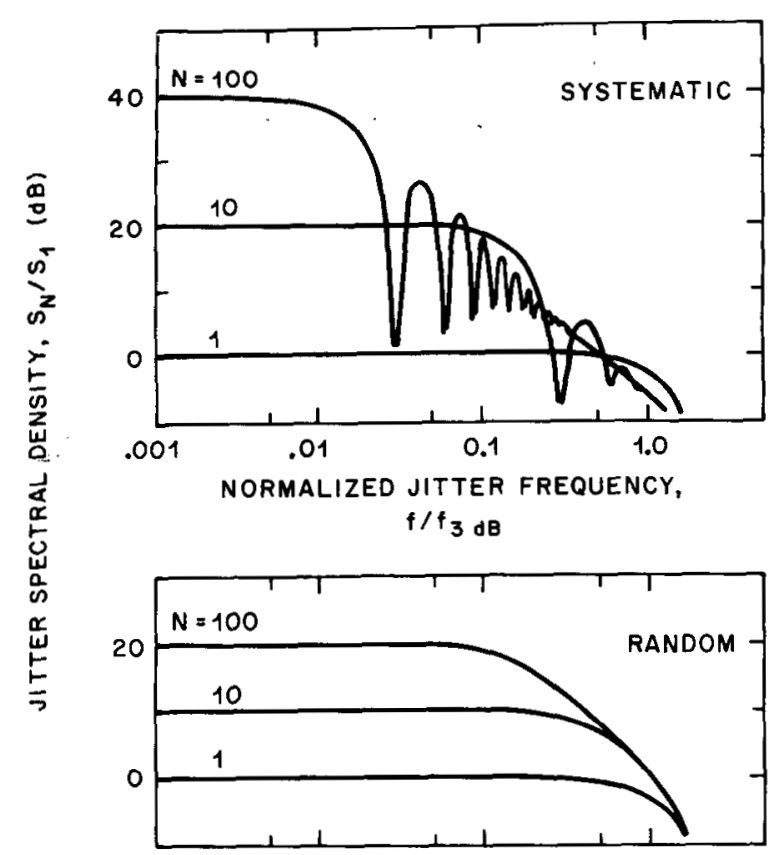

(a)

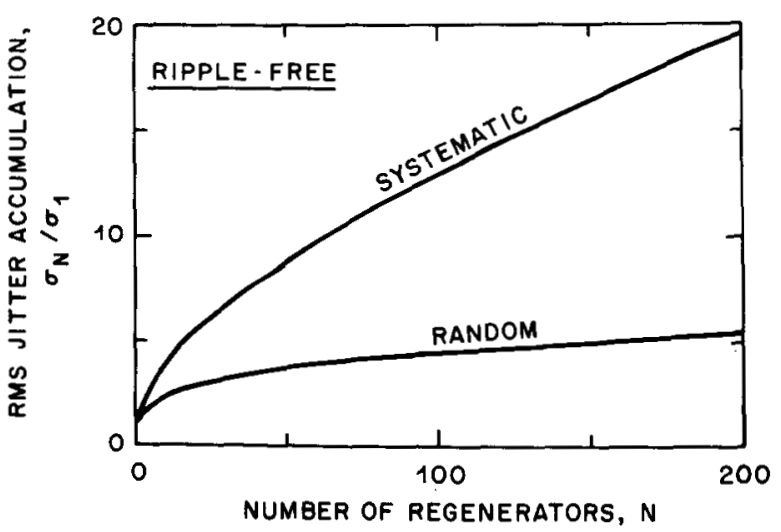

(c)

Fig. 7. (a) Systematic and (b) random jitter spectral densities for ripple-free transversal filters. The minima in the systematic spectra are caused by phase cancellations in the coherent sum of jitter transfer functions. (c) Rms jitter accumulation in the systematic and random cases. Both curves are normalized to $\sigma_{1}$, which is often much larger for systematic than for random jitter.

rms jitter accumulation curves for different amounts of Chebyshev ripple in a double-tuned filter. The ripple-free double-tuned filter with a $Q$ of 1200 has been selected as the approximate jitter equivalent of the ripple-free transversal filter with a $Q$ of 800 , considered below. Both filters have a center-frequency phase slope near $0.7^{\circ} / \mathrm{kHz}$.

\section{B. Effects of Transversal Filter Passband Ripple}

The jitter consequences of detuning ripple-free SAW transversal filters are not treated here. As in the case of ripplefree resonator filters, jitter peaking may be expected only when the detuning is well beyond permissible limits. The significant jitter-peaking mechanism with transversal filters is again passband ripple.

For reference purposes, Fig. 7 shows the jitter spectral density and the accumulation of rms systematic and random jitter for the ripple-free transveral filter case, computed from (10)-(12), (30), (31), and (4c). These results are substantially the same as in the familiar case of single-tuned resonator filters [9]. 
Analogous results are shown in Fig. 8 for the transversal filter model with a symmetric passband ripple of $0.1 \mathrm{~dB}$. The jitter spectral densities show an incipient systematic jitter peak at the $N=100$ stage, and a fully developed random jitter peak at the same stage. Evidently, the peak is partially' suppressed in the systematic case by coherent phase cancellations in (10). The impact of the above behavior on rms jitter accumulation is clear in Fig. 8(b). For the larger values of $N$, random jitter accumulates much faster than the systematic jitter, contrary to the ripple-free results in Fig. 7(b). It is reassuring that the normalization parameter $\sigma_{1}$ for random jitter is usually small enough to hold accumulation within reasonable bounds in transoceanic chains of regenerators having a small amount $(<0.05 \mathrm{~dB})$ of jitter peaking.

\section{Ripple Effects on Alignment Jitter}

The relative, or alignment, jitter at the last regenerator in a cascade of $N$ regenerators is quite sensitive to passband ripple. The rms alignment jitter has been computed from (15), (16), and (12) for the ripple-free filter described by (30) and (31), and for the ripply filters described by the "worstcase" forms of (4a) and (4b). Fig. 9 shows the results. Alignment jitter at the $N$ th regenerator is seen to change over from the traditional nonaccumulating behavior [9], [13] to a rapidly accumulating behavior as the ripple increases. Since any increase of relative jitter can raise the fraction of digital transmission errors, the ripple must be carefully limited to avoid degradation of system performance. Acceptable limits must be determined by the designer in allocating margins for the decision "eye-diagram."

\section{SUMmary AND DISCUSSION}

$\dot{W}$ e have investigated analytically the worst case effects of SAW filter passband ripple and detuning on jitter accumulation and alignment jitter in a cascade of digital regenerators. These matters are of current interest because passband ripple is nearly impossible to eliminate from a population of SAW transversal filters of simple design and manufacture, and because earlier work [12] had left unquantified the jitter effects of detuning. Results indicate the following.

1) Filter detuning by itself is not a practical hazard to jitter performance, since the detuning threshold for jitter peaking is larger than the threshold for copious digital decision errors [2]. Even the latter threshold must be avoided by appropriate system design.

2) Passband ripple will produce a form of jitter peaking, with the resultant tendency toward exponential jitter growth in a long regenerator cascade.

3) In the presence of jitter peaking, exponential jitter accumulation is much more pronounced for random jitter than for systematic jitter, contrary to the traditional behavior with ripple-free filters. The exponential growth of systematic jitter tends to be suppressed by phase coherence effects among the cascaded regenerators.

4) Although the coherence effects may be partially lost when the transversal filters show a random scatter in group delays, the amount of scatter in typical filter populations is too limited to result in a significant jitter increase from that cause alone.

5) Alignment jitter also can show runaway accumulation behavior in the presence of passband ripple, even though ripple-free transversal filters show the nonaccumulating behavior of single-tuned resonator filters [9].

All of the above findings are clearly important in the design of currently emerging transoceanic fiber systems, which use very long regenerator cascades. It should be noted that there are also important results for shorter undersea systems and for terrestrial long-haul fiber systems, where maintenance spans will seldom utilize more than 50 regenerators. For $N \leqslant$

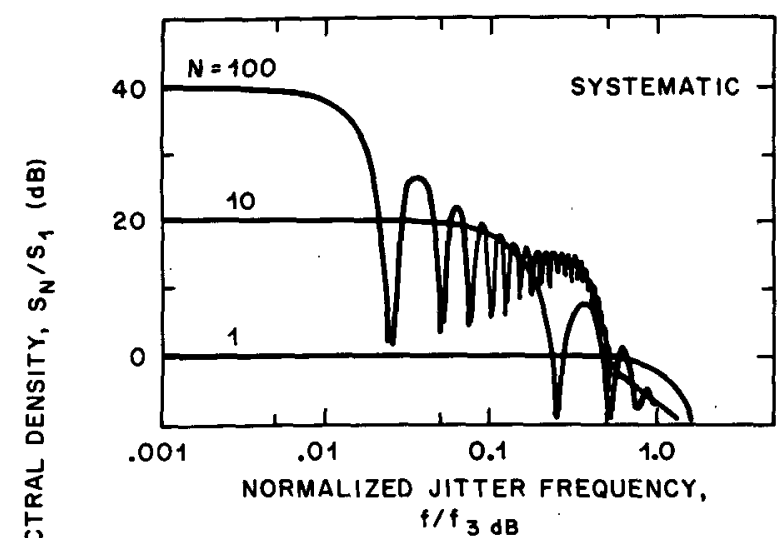

(a)

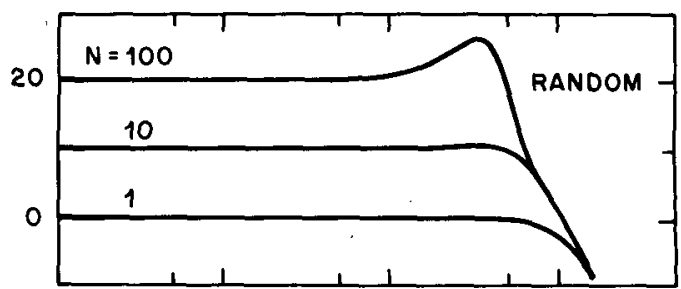

(b)

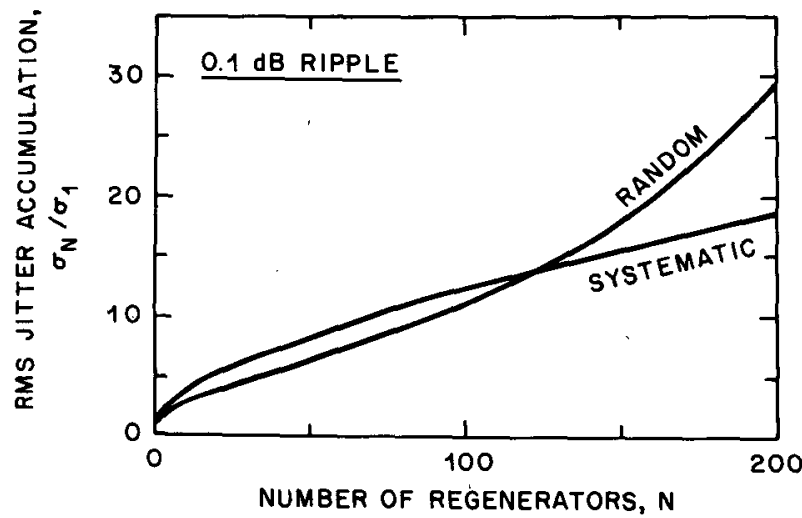

(c)

Fig. 8. (a) Systematic and (b) random jitter spectral densities for SAW transversal filters with $0.1 \mathrm{~dB}$ symmetric ripple. The large spectral components near $f / f_{3 \mathrm{~dB}}=0.3$ represent the effects of jitter peaking. (c) Associated rms jitter accumulation. Runaway systematic jitter growth has not set in up to $N=200$ because the corresponding spectral peak does not yet dominate the area under the curve.

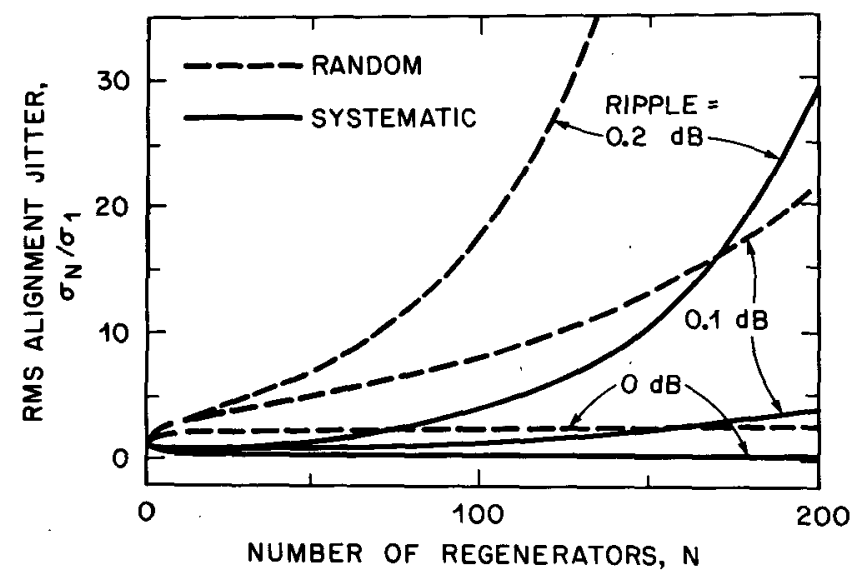

Fig. 9. Systematic and random alignment jitter with all regenerators having the specified values of jitter peaking. 
50, Figs. 5(b) and 8(b) show that there can be no harmful effects on jitter accumulation from filter detuning or from passband ripple below $0.1 \mathrm{~dB}$. However, Fig. 9 shows that ripple up to $0.2 \mathrm{~dB}$ could have a significant effect on alignment jitter for $N \leqslant 50$.

As we have emphasized throughout, the present results evaluate worst case situations, in which passband ripple has its most harmful effects. We should note, however, that a population of SAW filters will have varying passband asymmetries and a substantial spread in jitter-peak frequencies. Both kinds of randomness will tend to reduce and smear out the constructive interference effects of peaking along a regenerator cascade. It may therefore be expected that statistical variations among SAW retiming filters and the circuits in which they are imbedded will be beneficial on balance. Statistical effects have been studied [10], [14] since the completion of the present work, and have been found capable of strong suppression of the worst effects of jitter peaking.

\section{APPENDIX}

SAW transversal filters are characterized here by the simplest model that has the qualitative features of primary interest. With reference to Fig. 10, these features include the following.

a) The frequency dependence of the piezoelectric response of the transducers across the filter passband and neighboring sidebands. The sidebands are included to allow an estimate of jitter contributions passed in the sidebands.

b) The effect of transducer terminations on the filter response. Since the passband shape is sensitive to the terminations, they must be properly accounted for.

c) Perturbations of the transfer function caused by multiple reflections between the transducers. The most important reflections are the "triple-transit" signals illustrated in Fig. 10(b). Triple-transit signals interfere with the first-transit signals and thereby distort the passband.

Item c) causes the ripple of interest here. A filter transfer function incorporating all the desired features can be constructed with the help of a scattering formalism and the following assumptions.

1) The filter consists of a weak piezoelectric substrate (quartz for temperature stability) bearing a pair of identical interdigitated transducers with uniformly spaced electrodes and constant "tap weights," just as shown in Fig. 10. For the fractional bandwidths of interest $\left(Q^{-1} \equiv \Delta f / f_{c} \sim 10^{-3}\right.$, $f_{c}=$ center frequency, $\Delta f=$ full $3 \mathrm{~dB}$ bandwidth), each transducer will have hundreds of fingers.

2) The input and output transducers are separated by about 20 wavelengths. (For transoceanic systems, the separation should be small as possible to hold down the filter phase slope [2], but large enough to give effective RF isolation.)

3 ) The equivalent circuit of a transducer is that of a simple crossed-field model [15], [3], consisting of the static capacitance $C$ of the transducer, shunted by the dynamic (piezoelectric) conductance and susceptance of the interdigital structure; see Fig. 11.

4) A shunt inductance $\dot{L}$ is postulated in the electrical termination of each transducer to tune out $C$ at the filter center frequency $f_{c}$ (Fig. 11). Since the dynamic susceptance will also vanish at $f_{c}$ (see below), the input admittance effectively becomes a pure conductance at $f_{c}$.

5) The transducer conductance at $f_{c}$ is regarded as adjustable. This assumption is consistent with design options, and allows us to vary the conductance mismatch with respect to a given termination conductance, e.g., $(50 \Omega)^{-1}$. The conductance mismatch at $f_{c}$ controls the filter insertion loss, but more importantly for present purposes, it also controls the magnitude of the passband ripple.

With the above assumptions, and with the scattering diagram shown in Fig. 12, we can use standard scattering methods

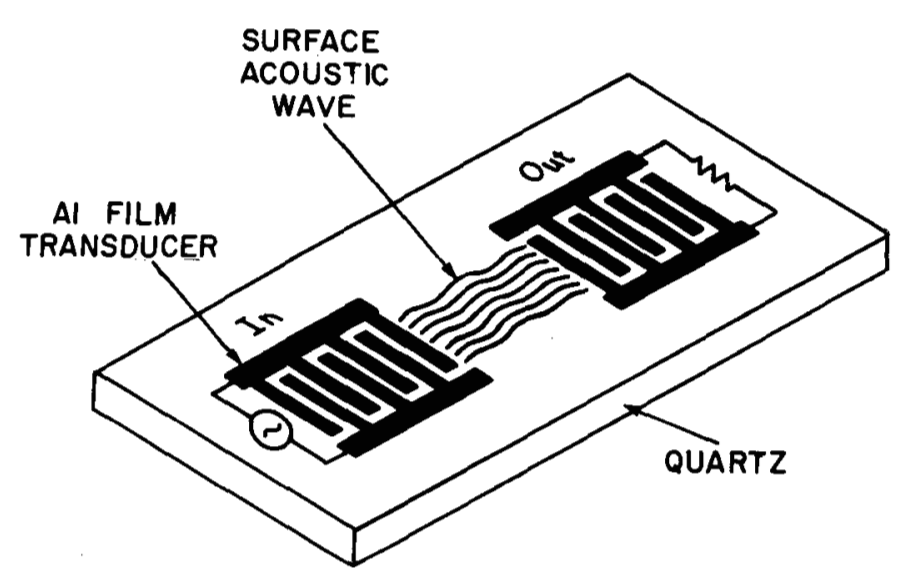

(a)

(1) FIRST TRANSIT

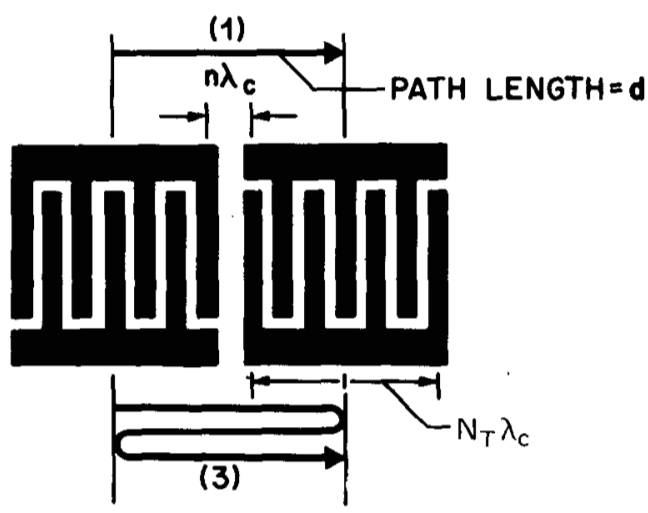

(3) TRIPLE TRANSIT

(b)

Fig. 10. (a) Sketch of the simplest type of SAW transversal filter, consisting of a piezoelectric crystalline substrate bearing two metal-film interdigital transducers for launching and receiving surface acoustic waves via electromechanical conversion processes. (b) Illustration of a triple-transit (twice-reflected) signal, path (3), which can interfere coherently with the first-transit (direct) signal, path (1), to cause a rippled frequency response. Also defined are $N_{T}$, the number of periods spanned by a transducer at the acoustic wavelength $\lambda_{c}$ of the filter center frequency, and $n$, the (arbitrary) number of wavelengths $\lambda_{c}$ between transducers.

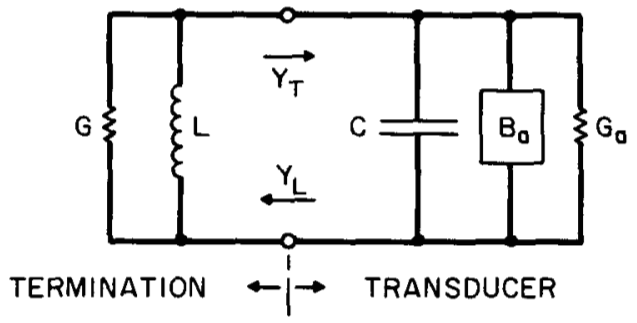

Fig. 11. Equivalent circuit of a simple SAW transducer, as given by the crossed-field model. The shunt inductor in the termination has been chosen for analytical simplicity in obtaining a conjugate susceptance match at $f_{c} . Y_{T}$ and $Y_{L}$ are the admittances looking into the transducer and into its termination, respectively. $G_{a}$ and $B_{a}$ are the dynamic conductance and susceptance of the transducer, $C$ is its static capacitance, and $G$ is the termination conductance. 


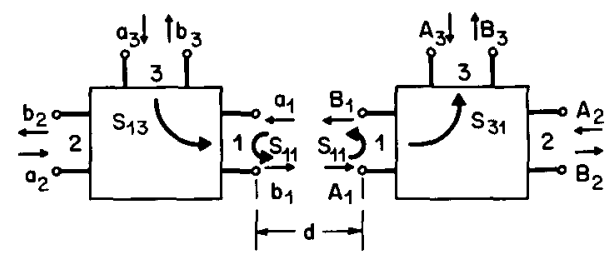

Fig. 12. Scattering diagram of the complete filter. Both transducers have the same scattering coefficients by assumption, with $S_{11}$ representing acoustic reflection, and $S_{13}=S_{31}$ representing electromechanical conversion. Input waves are represented by $\boldsymbol{a}_{j}$ or $\boldsymbol{A}_{j}$ and output waves by $\boldsymbol{b}_{i}$ or $\boldsymbol{B}_{i}$.

[6] to solve for the filter transfer function with multiple reflections, $\boldsymbol{H}(\omega) \equiv B_{3} / a_{3}$. The scattering boundary conditions used are $a_{2}=0=A_{2}$ (no acoustic inputs at the outer transducer ports), $A_{3}=0$ (no electrical input at the receiving transducer), and $a_{1}=t B_{1}$ and $A_{1}=t b_{1}$ (transmission relations), where $t$ is the propagation factor $t=\exp (-j \beta d), \beta=$ $2 \pi / \lambda, \lambda=$ acoustic wavelength. In terms of simple scattering parameters, one fïnds

$$
H=\frac{t S_{13}^{2}}{1-t^{2} S_{11}^{2}} .
$$

The numerator represents the direct signal path between the input $a_{3}$ and the output $B_{3}$, i.e., $S_{31} t S_{13}$. The denominator differs from unity because of multiple reflection effects, which are completely accounted for by the term $t^{2} S_{11}{ }^{2}$, where $S_{11}$ is the acoustic reflection coefficient at port 1 of each junction.

\section{Transfer Function Without Multiple Reflections}

Before we proceed to the general result, it is useful to examine the lowest order approximation to the transfer function,

$$
H_{0}=t S_{13}^{2}
$$

with reflection effects ignored. For the usual case of acoustically bidirectional transducers, which we have assumed here, the crossed-field transducer model yields [6]

$$
S_{13}^{2}=\frac{-2 G G_{a}}{\left(G+G_{a}+j b\right)^{2}}
$$

where the immittances may be identified with the help of Fig. 11:

$$
\begin{aligned}
Y_{T} & =G_{a}+j\left(B_{a}+\omega C\right) \\
& =\text { transducer input admittance } \\
Y_{L} & =G-j /(\omega L) \\
& =\text { termination admittance } \\
b & =B_{a}+\omega C-1 /(\omega L) .
\end{aligned}
$$

Subscript $a$ refers to dynamic acoustic immittances.

In the crossed-field model, the dynamic quantities are given by

$$
\begin{aligned}
& G_{a}(\omega) \approx G_{a}\left(\omega_{c}\right) E(x) \\
& B_{a}(\omega) \approx G_{a}\left(\omega_{c}\right) O(x) \\
& G_{a}\left(\omega_{c}\right) \approx \frac{4}{\pi} K^{2} N_{T} \omega_{c} C
\end{aligned}
$$

where $x, E(x), O(x), \omega_{c}$, and $N_{T}$ are defined at (4), and

$$
\begin{gathered}
K^{2}=\text { piezoelectric SAW coupling parameter } \\
(\approx 0.00116 \text { for ST }-\mathrm{X} \text { quartz }) .
\end{gathered}
$$

The forms are valid over the main filter passband and nearby sidebands when the filter $Q$ is high enough (e.g., $Q>100$ ). Since $\omega_{c}^{2} L C=1,(25)$ can also be written

$$
b=B_{a}+\frac{2 \omega_{c} C}{\pi N_{T}} x \approx B_{a}
$$

where the last approximation can be shown to be valid for present purposes. Finally, the propagation phase $\beta d$ can also be written as in (4c). Implicit in $\beta d$ is the convention that the equivalent "point" location of each transducer is its midpoint, with the scattering phases defined accordingly.

The lowest order transfer function $H_{0}$ is now explicitly given by (21), (22), (26)-(29), and (4c). In terms of the conductance mismatch parameter (5), the amplitude characteristic becomes

$$
|H(\omega)| \approx \frac{2 \mu E}{(1+\mu E)^{2}+(\mu O)^{2}}
$$

and the phase characteristic of the filter becomes

$$
\begin{aligned}
\phi(\omega) \approx & 2 \pi\left(N+n+\frac{1}{2}\right)+2\left(1+\frac{n}{N}\right) x \\
& +2 \tan ^{-1}\left(\frac{\mu O}{1+\mu E}\right) .
\end{aligned}
$$

The linear second term of (31) dominates the group delay of the filter, while the nonlinear last term is a minor contribution from the transducer scattering phases. Equation (30) provides us with the nominal filter insertion loss (not including internal losses) and allows us to evaluate the lowest-order (ripple-free) "filter $Q$." The nominal insertion loss is

$$
\begin{aligned}
I L & =-10 \log \left|H(\omega)^{2}\right|_{x=0} \\
& =-10 \log \left(\frac{4 \mu^{2}}{(1+\mu)^{4}}\right) .
\end{aligned}
$$

When $\mu$ is $\ll 1$, the $3 \mathrm{~dB}$ bandwidth may be found from the numerator of (30). The $3 \mathrm{~dB}$ points of $E$ occur where $x=$ $\pm 1 \mathrm{rad}$, so that

$$
Q \approx \frac{\pi}{2} N, \quad \mu \ll i
$$

As $\mu$ increases toward 1 , the frequency dependence of the denominator of (30) must be taken into account. Over the practical range $I L<8 \mathrm{~dB}$, available with bidirectional transducers, a more accurate approximation is given by

$$
N_{T}=\frac{2}{\pi} Q y(\mu)
$$

where $y(\mu)$ is the solution of the transcendental equation

$$
\begin{gathered}
(\mu E+1-a)^{2}+(\mu O)^{2}=a^{2}-2 a, \\
a=\frac{(1+\mu)^{2}}{\sqrt{2} \mu} .
\end{gathered}
$$


The right-hand side of (34), rounded to the nearest integer, gives the number of finger pairs in each transducer required to produce a specified ripple-free filter $Q$.

The variation of $y(\mu)=\pi N_{T} /(2 Q)$ with the conductance mismatch $\mu$ is shown in Fig. 2. The curve is an aid to the rapid parametrization of ripply transversal filters for jitter accumulation studies.

\section{Transfer Function with Ripple Present}

To account for multiple reflections, the full transfer function (20) must be utilized in place of (21). We therefore need the crossed-field expression for $S_{11}{ }^{2}$ [6],

$$
S_{11}^{2}=\frac{G_{a}^{2}}{\left(G+G_{a}+j b\right)^{2}} .
$$

Substituting (36) and (22) into (20), we obtain

$$
H(\omega)=\frac{-2 t \frac{G_{a}}{G}}{\left(1+\frac{G_{a}+j b}{G}\right)^{2}-\left(t \frac{G_{a}}{G}\right)^{2}}
$$

This form is suitable for direct computer manipulation with relations (26), (27),..(29), (4c), and (5). All that remains is to complete the specification of parameters. For that purpose, we use the relations cited in this paragraph to obtain the following analytical expressions for the amplitude and phase transfer functions:

$$
\begin{aligned}
& |H(\omega)|=2 \mu E /\left(P^{2}+R^{2}\right)^{1 / 2} \\
& \phi(\omega)=\pi+\beta d+\tan ^{-1}(R / P)
\end{aligned}
$$

where

$$
\begin{aligned}
& P=(1+\mu E)^{2}-(\mu O)^{2}-(\mu E)^{2} \cos (2 \beta d) \\
& R=2 \mu O(1+\mu E)+(\mu E)^{2} \sin (2 \beta d) .
\end{aligned}
$$

The last term in each of (39a) and (39b) carries the complexity added by the ripple.

It is instructive to compare the center frequency value of $\left|H\left(\omega_{c}\right)\right|^{2}$ obtained from (38a) with the lowest order value $\left|H_{0}\left(\omega_{c}\right)\right|^{2}$, obtained from (30). With the help of (26), (27), (29), (5), and some algebra, we find

$$
\begin{aligned}
\rho & \equiv\left|\frac{H\left(\omega_{c}\right)}{H_{0}\left(\omega_{c}\right)}\right|^{2} \\
& =\frac{(1+\mu)^{4}}{\left[(1+\mu)^{2}-\mu^{2}\right]^{2}+4 \mu^{2}(1+\mu)^{2} \sin ^{2}\left(\beta_{c} d\right)} .
\end{aligned}
$$

This ratio is $<1$ when $\sin ^{2}\left(\beta_{c} d\right)=1$ and $>1$ when $\sin ^{2}\left(\beta_{c} d\right)=$ 0 . Thus, the model may either increase or decrease the center frequency response in accord with the choice of transducer separation parameter $n$ [see (4c)]. The extrema are given by

$$
\rho_{\text {extrema }}=\left(1 \pm \frac{\mu^{2}}{1+\mu^{2}}\right)^{-2}
$$

The alternatives are precisely what we expect when the triple-transit signal interferes with the direct-transit signal. The interference may be either constructive or destructive, depending upon the extra phase shift of the triple-transit signal. A diminished response at center frequency implies a dip at passband center, and computer simulation confirms this behavior, as illustrated in Fig. 3(c). We only have to choose

$$
N_{T}+n=\text { integer } \pm 1 / 4
$$

to obtain the deepest central dip. With $n=20 \pm 0.25$, simulation yields the relationship shown in Fig. 2 between the ripple and the conductance mismatch parameter $\mu$.

To recapitulate, the model parameters implicit in (38) have been found in accord with the following flow diagram:

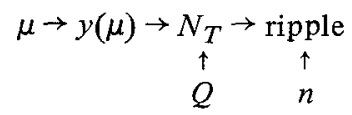

where $Q$ and $n$ were given preselected values. Repeated application of this diagram for different values of $\mu$ leads to Fig.' 2 , which can be used, for example, to determine $N_{T}$ for preselected values of ripple, $Q$, and $n$. For very small values of $n / N_{T}$, the ripple is quite insensitive to $N_{T}$ (but remains sensitive to $n$ ). In that case, the ripple is also insensitive to $Q$, since $N_{T}$ scales linearly with $Q$. The curves of Fig. 2 correspond to the insensitive case, so that Fig. 2 is actually valid for all $Q$ 's larger than the 800 value used for computation.

The situation considered above, with symmetric ripple and a minimum at passband center, is the worst case from the standpoint of jitter accumulation. The opposite extreme, where transmission near $\omega_{c}$ is symmetrically enhanced by the triple-transit signal, could conceivably lead to jitter accumulation under special conditions, but not as much. Between the extremes (e.g., $20<n<20.25$ ), the passband distortion will be partly antisymmetric about the center frequency, as shown in Fig. 3(a). The antisymmetric part does not contribute to jitter peaking [10]

\section{ACKNOWLEDGMENT'}

We are indebted to $P$. R. Trischitta for experimental information that served as a crosscheck in the course of this work.

\section{REFERENCES}

[1] R. J. Murray and P. D. White, "SAW components answer today's signal processing needs," Electronics, vol, 8, pp. 120-124, Sept. 1981.

[2] R. L. Rosenberg, D. G. Ross, P. R. Trischitta, D. A. Fishman, and C. B. Armitage, "Optical fiber repeatered transmission system utilizing SAW filters," IEEE Trans. Sonics Ultrason., vol. SU-30, pp. 119$126,1983$.

[3] R. L. Rosenberg and L. A. Coldren, "Broader-band transducercoupled SAW resonator filters with a single critical masking step," in IEEE Ultrason. Symp. Proc., 1980, pp. 164-168.

[4] E. Rosa, "Analysis of phase-locked timing extraction circuits for pulse code modulation," IEEE Trans. Commun., vol. COM-22, pp. 12361249, 1974.

[5] J. Wu and E. L. Varma, "Analysis of jitter accumulation in a chain of digital regenerators," in Proc. IEEE GLOBECOM, 1982, vol. II, pp. 653-657.

[6] R. L. Rosenberg and L. A. Coldren, "Scattering analysis and design of SAW resonator filters," IEEE Trans. Sonics Ultrason., vol. SU-26, pp. 205-230, 1979.

[7] Reference Data for Radio Engineers, 6th ed. ITT, Sect. 9.

[8] A. J. Slobodnick, Jr., T. L. Szabo, and K. R. Laker, "Miniature SAW filters," Proc. IEEE, vol. 67, pp. 129-146, 1979.

[9] C. J. Byrne, B. J. Karafin, and D. B. Robinson, Jr., "Systematic jitter in a chain of digital regenerators," Bell Syst. Tech. J., vol. 42, pp. 2679-2714, 1963. 
[10] C. Chamzas, "Accumulation of jitter: A stochastic model," $A T \& T$ Bell Lab. Tech. J., vol. 64, pp. 43-76, 1985.

[11] H. E. Rowe, "Timing in a long chain of regenerative binary repeaters," Bell Syst. Tech. J., vol. 37, pp. 1501-1542, 1958.

[12] V. Mengali and G. Pirani, "Jitter accumulation in PAM systems," IEEE Trans. Commun., vol. COM-28, pp. 1172-1183, 1980.

[13] J. M. Manley, "The generation and accumulation of timing noise in PCM systems-An experimental and theoretical study," Bell Syst. Tech. J., vol. 48, pp. 541-613, 1969.

[14] R. L. Rosenberg, C. Chamzas, and D. A. Fishman, "Timing recovery with SAW transversal filters in regenerators of undersea long-haul fiber transmission systems," J. Lightwave Technol., Special Issue on Undersea Lightwave Communications, vol. LT-2, pp. 917-925, Dec. 1984.

[15] W. R. Smith et al., "Analysis of interdigital surface-wave transducers by use of an equivalent circuit model," IEEE Trans. Microwave Theory Tech., vol. MTT-17, pp. 856-864, 1969.

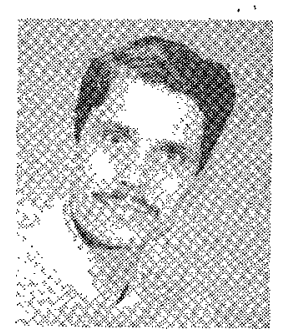

Daniel A. Fishman was born in New York, NY, on September 17, 1955. He received the B.S. degree (summa cum laude) in physics from the City College of New York, New York, in 1978, and the S.M. degree from the Massachusetts Institute of Technology, Cambridge, in 1981.

He has worked in the fields of fiber optics, electrodynamics applied to electronic countermeasures, and magnetostatics at various institutions. Since completing his thesis at M.I.T., he has been with AT\&T Bell Laboratories, Holmdel, NJ, where he is a member of the Technical Staff in the Long Haul Lightwave System Development Department. His current responsibilities include laser transmitter testing and evaluation, microwave circuit design, and jitter simulation and analysis.

Mr. Fishman is a member of Phi Beta Kappa and the American Physical Society, and is an associate member of Sigma Xi. He was the recipient of the C.C.N.Y. 1978 Ward Medal in physics.

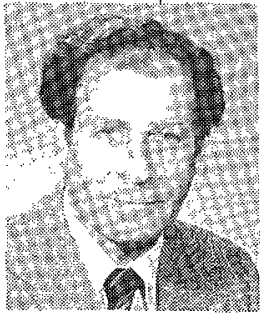

Robert L. Rosenberg (M'73-SM'80) was born in Passaic, NJ, on April 10, 1924. He received the S.B. degree in electronic physics from Harvard University, Cambridge, MA, in 1946, and the M.S. and $\mathrm{Ph} . \mathrm{D}$. degrees in physics from Syracuse University, Syracuse, NY, in 1952 and 1957, respectively.

He joined the Research Division of Bell Laboratories in 1955, and has worked in the fields of semiconductors, magnetics, lasers and optics, and ultrasonics. He is coinventor of the "jet-stream" dye laser. He is currently a Distinguished Member of the Technical Staff, Transmission Systems Division, AT\&T Bell Laboratories, Holmdel, NJ, with system and consulting responsibilities in the SAW timing-recovery filter program for undersea and other digital optical fiber transmission systems.

Dr. Rosenberg was a corecipient of the 1979 Best Paper Award of the IEEE Group on Sonics and Ultrasonics, and he serves on the Technical Program Committee of the Ultrasonics Symposium. He is a member of the American Physical Society, Sigma Xi, and Sigma Pi Sigma.

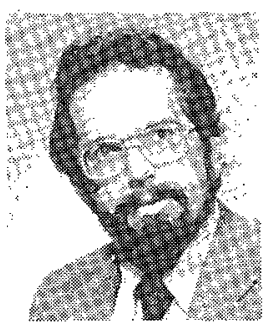

Christodoulos Chamzas (S'75-M'79) was born in Komotini, Greece, in 1951 . He received the $\mathrm{Di}$ ploma in electrical and mechanical engineering from the National Technical University of Greece, Athens, in 1974, and the M.S. and Ph.D. degrees in electrical engineering from the Polytechnic Institute of New York, Farmingdale; NY.

From 1979 to 1982 he was an Assistant Professor with the Department of Electrical Engineering, Polytechnic Institute of New York, where he is currently an Adjunct Associate Professor. Since 1982 he has been with AT\&T Bell Laboratories, Holmdel, NJ. His primary interests are in signal processing and communication systems. He has held summer positions in Greece, Portugal, and England as well as at Bell Laboratories. $\mathrm{Xi}$.
Dr. Chamzas is a member of the Technical Chamber of Greece and Sigma 